\title{
Cancer Risk Assessment: Optimizing Human Health Through Linear Dose-Response
}

\section{Models}

Edward J. Calabrese, Ph.D. ${ }^{*}$, Professor of Toxicology, School of Public Health \& Health Sciences, Department of Environmental Health Sciences, Morrill I N344, University of Massachusetts, Amherst MA 01003, Phone: 413-545-3164, Fax: 413-545-4692, E-mail: edwardc@schoolph.umass.edu

Dima Yazji Shamoun, Ph.D., Mercatus Center, George Mason University, 3434 Washington Blvd., Arlington VA 22201, Phone: 703-220-7308, E-mail:dshamoun@ mercatus.gmu.edu

Jaap C. Hanekamp, Ph.D., Roosevelt Academy, Lange Noordstraat 1, NL-4331 CB Middelburg, The Netherlands, Phone: 31-79-346-0304, E-mail: j.hanekamp@roac.nl

*Corresponding Author 


\begin{abstract}
This paper proposes that generic cancer risk assessments be based on the integration of the Linear Non-Threshold (LNT) and hormetic dose-responses since optimal hormetic beneficial responses are estimated to occur at the dose associated with a $10^{-4}$ risk level based on the use of a LNT model as applied to animal cancer studies. The adoption of the $10^{-4}$ risk estimate provides a theoretical and practical integration of two competing risk assessment models whose predictions cannot be validated in human population studies or with standard chronic animal bioassay data. This model-integration reveals both substantial protection of the population from cancer effects (i.e. functional utility of the LNT model) while offering the possibility of significant reductions in cancer incidence should the hormetic dose-response model predictions be correct. The dose yielding the $10^{-4}$ cancer risk therefore yields the optimized toxicologically based "regulatory sweet spot".
\end{abstract}

Key Words: risk assessment, dose-response, hormesis, linearity, LNT, precautionary principle

\title{
1. Introduction
}

The assessment of cancer risks from exposure to ionizing radiation and chemical carcinogens by regulatory agencies worldwide is typically performed via the use of linear at low dose modeling. The linear non-threshold (LNT) approach for cancer risk assessment was first proposed for cancer risk assessment by the U.S. National Committee for Radiation Protection and Measurement (NCRPM) in 1958, following the recommendation of the U.S. National Academy of Sciences (NAS) Biological Effects of Atomic Radiation (BEAR) I Genetics Panel to 
switch from a threshold to a linear model for assessing genomic risk from ionizing radiation in $1956^{1,2}$.

The LNT approach was later adopted by regulatory agencies starting in the late 1970s assessing risks for chemical carcinogens in all media (e.g. air, water, food and soil) ${ }^{3}$. The initial transition from the threshold to the LNT approach in the mid more 1950s was made prior to the discovery of DNA repair, adaptive responses with chemical mutagens and ionizing radiation, apoptosis, pre-conditioning and the resurgence of the hormetic concept, all of which could affect the shape of the dose response in the low-dose zone. The clarification of different mechanisms of action for carcinogens has encouraged the development of cancer risk assessment methods that incorporate knowledge of species specificity and threshold. These approaches are often employed by the U.S. EPA and FDA and most European authorities for non-genotoxic carcinogens $^{4-7}$.

These developments have challenged the theoretical and mechanistic basis of the LNT, along with the recognition that epidemiological methods are in effect not capable of detecting risks below twice the normal background ${ }^{8}$. Furthermore, the massive mega-mouse study that used 24,000 animals was only able to estimate risk at the $1 \%$ level (ED01 study) ${ }^{9}$. Similar limitations were reported for a cancer bioassay study with >40,000 trout (Bailey et al., 2009) ${ }^{10}$. These methodological limitations along with the more recent developmental insights on the plethora of adaptive mechanisms that act at low doses have revealed limitations of the LNT model. 


\section{Developments}

The dose-response model that has been shown to have biological plausibility, especially in the low dose zone, is hormesis, a biphasic dose-response. Current interest in hormesis can be tracted back to the research of Thomas Luckey on radiation hormesis ${ }^{11}$ and on chemical hormesis by Tony Stebbing ${ }^{12}$. These researchers stimulated the electric power utilities of Japan and the U.S. to conduct the first hormesis conference in August, 1985. These three events reactivated interest in the hormesis concept.

Since the initial hormesis conference mentioned here, multiple books have been published on hormesis ${ }^{13-21}$. Also, many chapters on hormesis in toxicology and pharmacology texts have been produced; hormesis has been the focus of more than a dozen conferences; multiple symposia at major society meetings have addressed hormesis. It is the subject of more than 2,000 scientific publications in peer-reviewed journals, and the object of more than 30,000 citations in the Web of Science/Knowledge. Extensive documentation of hormetic dose responses have been summarized from a large and continuously updated database. ${ }^{22-24}$

The hormetic dose-response was also found to make more accurate predictions than the LNT or threshold dose-response models in head-to-head comparisons using large, independent data sets $^{25-27}$. Detailed mechanisms of 400 hormetic dose responses have recently been summarized $^{28}$. Additionally, the hormetic dose response therefore has been demonstrated to be highly generalizable, being independent of biological model (i.e., phylogenetically diverse-from bacteria to humans; in vitro and in vivo), level of biological organization (i.e., cell, organ and organism), endpoint, inducing agent and mechanism. 


\section{Objective - Integration}

Based on these features, it has been proposed that the hormetic dose-response should become the default model for risk assessment for both carcinogens and non-carcinogens. The hormesis database provides strong evidence that dose-response relationships for carcinogens (e.g., DDT, dioxin, multiple PAHs, ionizing radiation) and non-carcinogens typically display hormetic dose response patterns with similar quantitative features. While this line of argument has been made ${ }^{29}$, this is not the purpose of this paper. The present paper proposes a "practical" and straightforward harmonization of both the LNT and hormetic models for cancer risk assessment. As is customary in such convergences, common ground is sought by various entities (e.g., regulatory agencies and regulated industries), while differences are still recognized and will remain unresolved for now.

We see the following reasons why integration of both models would be beneficial. First, if hormesis describes low-dose exposure impacts of chemicals/ionizing radiation more accurately than the LNT-model does, then the regulatory authorities should apply the best the toxicological sciences have to offer. The hormetic dose response requires rigorous study designs in order to be properly evaluated, with large numbers of doses, with proper dose spacing, and often within a dose-time framework. When such data are available, the hormetic dose response has far outperformed the threshold and linearity dose response model for accuracy in estimating low dose effects. ${ }^{25-27}$

Second, considering the developments in analytical chemistry, increasingly lower levels of chemicals can be detected. We have entered the realm of atto- (part per quintillion; $10^{-18}$ ) and zeptomoles (part per sextillion; $10^{-21}$ ) of detectable analytes ${ }^{30}$. Consequently, the unspoken 'logic' of the LNT-model infers that a 'clean bill of health' can never be truly given ${ }^{31}$. The 
technology-driven stringency of regulation in the context of the LNT-model can be attenuated with the aid of the biphasic dose-response model. As a result, regulatory expenditures will be reduced along with benefit optimization ${ }^{32}$.

Third, the biphasic dose-response model underscores the beneficial adaptability of organisms' responses to chemicals exposure, whereby regulation that expresses the functional integration of both the LNT and hormetic models is better able to address society's fears of carcinogen exposure.

\section{Integration - Roadmap}

How then do we envision this integration, that is, the harmonization of the hormesis and LNT dose response models for cancer risk assessment? The reconciliation of these two divergent models can surprisingly be made in a direct and uncomplicated fashion.

1) The key aspect of the hormesis/LNT convergence is that when risks are based on chronic animal bioassay studies, the optimal protective effects (i.e., reduction in tumor incidence for the affected below the control group) is predicted to occur at the same dose at which the LNT predicts $10^{-4}$ risk.

2) To achieve this value, the hormetic-based approach would first estimate a $1 \%$ response from the animal bioassay via a BMD-type methodology. When this derived-dose is divided by factor of 100 , it yields slightly less than a risk of $10^{-4}$. This was shown to be the case for ten highly diverse data sets by Gaylor ${ }^{33}$. The hormetic risk assessment methodology of Calabrese and $\mathrm{Cook}^{34}$, which is optimized at the same dose that the LNT estimates a $10^{-4}$ risk level, predicts benefit while the LNT estimates enhanced cancer risk. 
3) We propose that cancer risk assessment adopt an acceptable risk of $10^{-4}$ using the LNT model since this dose would also yield the optimal hormesis dose response benefit. This dose is the so-called regulatory "sweet-spot" that provides substantial protection against theoretical low dose risks that are far below the detection of even the most demanding epidemiological and toxicological studies/methods, while including benefits predicted by the hormetic dose response model (Figure 1). This approach would also have the significant societal benefit of affecting a profound reduction in costs (i.e., financial and predicted adverse health), markedly affecting cost/benefit analyses.

4) In a population of one million people, the $10^{-4}$ risk predicts 100 people (i.e., $10^{6}$ people $\mathrm{x}$ $10^{-4}$ risk $\left.=100\right)$ affected with an organ-specific cancer (e.g., lung, kidney, bladder, etc.) by some deleterious agent that is added to the background for cancer of that organ (Figure 1). Assuming a 25\% tumor background incidence, 250,000 of the one million people would be predicted to develop tumors. If the organ in question was responsible for $5 \%$ (e.g., bladder) of the above $25 \%$ (i.e., 250,000 people), it would represent 12,500 of the 250,000 people with cancer (i.e., $0.25 \times 0.05=0.0125)\left(0.0125 \times 10^{6}=12,500\right)$. Many organ-specific tumors, including the bladder, affect about 3.5 to $6.0 \%$ of the tumor occurrence $^{35}$, thus the use of $5 \%$ for an organ like the bladder would be a reasonable expectation. Organs affecting a notably higher proportion of people (e.g., about 16-18\% per cancer type) are those cancers of the lung, breast and prostate. The 100 newly affected people with chemically induced bladder cancer are then randomly distributed amongst the entire population of one million. This suggests that $25 \%$ of the 100 will already be in the process of developing a background tumor, with about $5 \%$ of those already targeted for a "spontaneous" bladder tumor $(0.25 \times 0.05=1.25 \%)$. The net result 
of background (i.e. spontaneous) and tumor-induction via a chemical carcinogen at $10^{-4}$ is 12,500 ("background") plus 100 new chemically induced cases (i.e., 12,500 + $100=$ 12,600) minus 1 due to spontaneous and induced bladder tumors in the same individuals. This would yield a total of 12,599 individuals with bladder cancer. The hormetic benefit is likely to affect both background and induced tumor incidence, reducing their incidence by roughly $25 \%^{24}$, lowering the predicted total number of affected people $(12,500)$ by about 3,150 . There can be other situations in which the chemical may affect multiple organs with different tumor backgrounds and induced tumor incidence, affecting the nature and complexity of the assessment. For example, in the case of dioxin, it was shown in the Kociba et al. study ${ }^{36}$ that has been widely used for cancer risk assessment that hormetic effects appear to occur in multiple organs (i.e., Females: liver, ovary, uterus, cervix/vagina, mammary, pituitary and adrenal; Males: liver, pulmonary, pituitary, pancreas and adrenal). In such cases it may be possible to select that dose which displays the lowest overall tumor incidence for risk assessment purposes. In theory, this type of situation may be predicted to have a greater beneficial effect than described for the bladder cancer. However, it would not be unexpected for the optimal effect could vary by organ. Using a financial metaphor, the convergence of the LNT/10 ${ }^{-4}$ risk and hormesis methodologies permits the protecting of one's "principle" (i.e., impossible to detect chemically-induced increase in cancer risk) while adding considerable benefit (i.e., large reduction in cancer risk for those affected organs). This compromise strikes an optimized balance in which there is a very low theoretical risk increase and a very high theoretical benefit. Choosing a $10^{-6}$ acceptable risk would reduce 99 of the 100 
theoretically affected people while eliminating the possible hormetic benefit. This type of strategy would prevent the possibility of beneficial effects, which could be substantial.

5) The example presented above addresses the risk of a single complete carcinogen. However, humans live in a highly complex environment involving exposure to a vast array of complete carcinogens, tumor promoters, chemoprotective chemicals and physical agents, all superimposed on dynamic metabolic processes, numerous adaptive mechanisms and complex exposure dynamics. Predicting cancer incidence of complex mixtures from experimental and epidemiological studies is problematic, if not impossible. A very limited, simplified and yet mechanistically oriented approach to assess complex carcinogenic mixtures is the toxic equivalent factor (TEF) that assumes additive processes that act identically (e.g. same receptor) for similarly grouped agents (e.g. dioxins, PAHs and PCBs). The TEF concept was integrated within a LNT perspective. Epidemiological evaluations of complex mixtures reveal the failure of predictions of animal studies to predict human responses. For example, a cup of coffee contains $>1000$ chemicals of which approximately 30 have been tested for cancer. Of these the majority were carcinogenic in standard rodent model testing. Each cup of coffee contains $>10 \mathrm{mg}$ of rodent carcinogens, with American adults drinking three cups per day ${ }^{37,38}$. The situation gets more complex as more carcinogens are added via the roasting process. However, despite such exposures to natural and roasted process-related carcinogens, comprehensive epidemiological studies reveal neutral or beneficial effects from lifetime coffee drinking depending on the organ ${ }^{39,40}$. Thirty-two occupational epidemiological studies (i.e. case-control-19 studies; cohort-13 studies) of gasoline exposure which is a highly complex and variable mixture of $>500$ saturated/unsaturated hydrocarbons 
revealed no pattern or clear association between gasoline and any cancer ${ }^{41}$. Furthermore, dose responses of complex mixtures [e.g. petroleum ${ }^{42}$, waste-water treatment effluents ${ }^{43-}$ ${ }^{45}$, complex organochlorine mixtures ${ }^{46}$ over a broad dose response often conform to an hormetic dose response. These findings support the conclusion that complex mixtures and induce hormetic dose responses and can be evaluated within the framework proposed here.

6) An important implication of model uncertainty is that it has the potential to undermine and challenge the use of LNT in toxic tort litigation cases. The acknowledgement of substantial and unresolved uncertainty in risk assessment may preclude causation judgments with low dose exposures. In fact, the use of LNT in toxic tort cases in the United States has been successfully challenged in numerous litigations affecting ionizing radiation, asbestos as well as chemical carcinogens, principally due to its lack of validation capacity, inconsistency with published findings and the recognition of substantial adaptive mechanisms that undermine an LNT interpretation ${ }^{47-49}$.

\section{Discussion}

The search for public health common ground via the integration of opposing risk assessment models is a new approach in the process of risk assessment harmonization. It permits the strengths of opposing perspectives to be incorporated into a unified risk assessment approach. It is recognized that estimates of low risk is a speculative activity, especially when the data are derived from high dose toxicology studies and that there is no current practical way around this limitation. The present recommendation is viewed as substantially conservative, creating the 
opportunity to benefit from the induction of adaptive responses while recognizing and incorporating model uncertainty into the risk assessment process. We believe that this is a sound foundation upon which to base environmental public health policy.

The precautionary principle, which is at the core of modern governmental environmental health policies, is founded on a toxicological assumption that lower is always safer/better and that zero exposure, especially for carcinogens, is the goal [maximum contaminant level goal (MCLG)] as seen for EPA drinking water standards. The precautionary principle was strongly influenced during its formative development by belief in LNT predictions. Harmonizing of the LNT and hormesis dose response models can provide a vehicle not only for cancer risk assessment but also a novel means, along with a more biologically based foundation, to guide a broad range applications of the precautionary principle. 


\section{Acknowledgement}

Long-term research activities in the area of dose response have been supported by awards (to EJC) from the US Air Force (FA9550-13-1-0047) and ExxonMobil Foundation (S18200000000256) over a number of years. The U.S. Government is authorized to reproduce and distribute for governmental purposes notwithstanding any copyright notation thereon. The views and conclusions contained herein are those of the authors and should not be interpreted as necessarily representing policies or endorsement, either expressed or implied. Sponsors had no involvement in study design, collection, analysis, interpretation, writing and decision to submit. 


\section{References}

1. Whitemore, GF. The National Committee on Radiation Protection, 1928-1960: From Professional Guidelines to Government Regulation. Department of the History of Science, thesis dissertation. Harvard University, Cambridge MA, 1986).

2. Jolly, JC. Thresholds of uncertainty: Radiation and responsibility in the fallout Controversy. Dissertation, Oregon State University, Oregon (2003).

3. National Academy of Sciences (NAS). Drinking Water and Health (NAS, Washington DC, 1977) pp. 939.

4. Williams, GM. Mechanisms of chemical carcinogenesis and application to human cancer risk assessment. Toxicology 166, 3-10 (2001).

5. Williams, GM, Iatropoulos, MJ, Jeffrey, AM. Dose-effect relationships for DNA-reactive liver carcinogens, Chapter 12. In: The Cellular Response to the Genotoxic Insult: The Question of Threshold for Genotoxic Carcinogens (H. Greim, RJ Albertini, Editors). The Royal Society of Chemistry: London, UK (2012).

6. Page, NP, Singh, DV, Farland, W, Goodman, JL, Conolly, RB, et al. Implementation of EPA revised cancer assessment guidelines: Incorporation of mechanistic and pharmacokinetic data. Fundam. Appl. Toxicol. 37, 16-36 (1997).

7. Whysner, J, Williams, GM. International cancer risk assessment: The impact of biologic mechanisms. Reg. Toxicol. Pharmacol. 15, 41-50 (1992).

8. Taubes, G. Epidemiology faces its limits. Science 269, 164-169 (1995).

9. Bruce, RD, Carlton WW, Ferber, K H, Hughes, DH, Quast, J F, et al.(Members of the Society of Toxicology ED01 Task Force). Re-examination of the ED01 study: Adjusting for time on study. Fund. Appl. Toxicol. 1, 67-80 1981).

10. Bailey, GS, Reddy, AP, Pereira, CB, Harttig, U, Baird, W, et al. Non-linear cancer response at ultra-low dose: a 40,800-animal ED001 tumor and biomarker study. Chem. Res. Toxicol. 22, 1264-1276 (2009).

11. Luckey, TD. Ionizing Radiation and Hormesis (CRC Press, FL, 1980).

12. Stebbing, ARD. Hormesis - the stimulation of growth by low levels of inhibitors. Sci. Total Environ. 22, 213-234 (1982).

13. Luckey, TD. Radiation Hormesis. Oxidative Stress and Hormesis in Evolutionary Ecology and Physiology: A Marriage Between Mechanistic and Evolutionary Approach (CRC Press, Florida, 1992). 
14. Elliott K. Is a Little Pollution Good For You? (Oxford University Press, New York, 2008) $264 \mathrm{p}$.

15. Mattson, MP, Calabrese, EJ. Hormesis: A Revolution in Biology, Toxicology and Medicine. . (Humana Press Inc., 2010).

16. Stebbing, T. A Cybernetic View of Biological Growth: The Maia Hypothesis (Cambridge University Press, New York, 2011).

17. Costantini, D. Oxidative Stress and Hormesis in Evolutionary Ecology and Physiology: A Marriage Between Mechanistic and Evolutionary Approaches (Springer, New York, 2014).

18. Rattan, SIS, LeBourg, E. Hormesis in Health and Disease. (CRC Press, Florida, 2014).

19. Sanders, CL. Radiation Hormesis and the Linear-No-Threshold Assumption (Springer, New York, 2010).

20. Calabrese, E J. Biological Effects of Low Level Exposures to Chemicals and Radiation (Lewis Publishers, Michigan, 1992).

21. Calabrese, EJ. Biological Effects of Low Level Exposures: Dose-Response Relationships (CRC Press, Florida, 1994).

22. Calabrese, EJ, Blain, R. The occurrence of hormetic dose responses in the toxicological literature, the hormesis database: An overview. Toxicol Appl. Pharmacol. 202, 289-301 (2005).

23. Calabrese, EJ, Blain, RB. Hormesis and plant biology. Environ. Poll. 157, $42-48$ (2009).

24. Calabrese, EJ, Blain, RB. The hormesis database: The occurrence of hormetic dose responses in the toxicological literature. Reg. Toxicol. Pharmacol. 61, 73-81 (2011).

25. Calabrese, EJ, Baldwin, LA. The hormetic dose response model is more common than the threshold model in toxicology. Toxic. Sci. 71, 246-250 (2003).

26. Calabrese, E J, Staudenmayer, JW, Stanek, EJ, Hoffmann, G R, Hormesis outperforms threshold model in National Cancer Institute antitumor drug screening database. Toxicol. Sci. 94, 368-378 (2006).

27. Calabrese, EJ, Stanek, EJ, Nascarella, MA, Hoffmann, GR. Hormesis predicts low-dose responses better than threshold model. Intern. J. Toxicol. 27, 369-378 (2008).

28. Calabrese, EJ. Hormetic mechanisms. Crit. Rev. Toxicol. 43, 580-606 (2013).

29. Calabrese, EJ. Hormesis: from marginalization to mainstream: A case for hormesis as the default dose-response model in risk assessment. Toxicol. Appl. Pharmacol. 197, 125-136 (2004). 
30. Pagnotti, VS, Chubatyi, ND, McEwen, CN. Solvent Assisted Inlet Ionization: An Ultrasensitive New Liquid Introduction Ionization Method for Mass Spectrometry. Anal. Chem. 83, 3981-3985 (2011).

31. Hanekamp, JC, Kwakman, J, Pieterman, R, Ricci, PF. The administrative ordering of nature and society - precaution and food safety at the molecular and global level. Europ. J. Risk Reg. 3, 313-325 (2012).

32. Keeney, RL. Estimating Fatalities Induced by the Economic Costs of Regulations. J. Risk Uncert. 14, 5-23 (1997).

33. Gaylor, DW. Quantitative risk analysis for quantal reproductive and developmental effects. Environ. Health Perspect. 79, 243-246 (1989).

34. Calabrese, E J, Cook, RR. Hormesis: How it could affect the risk assessment process. Hum. Exper. Toxicol. 24, 265-270 (2005).

35. National Cancer Institute (NCI). SEER Stat Fact Sheets, (2014) http://seer.cancer.gov/statfacts/html/urinb/html.

36. Kociba, RJ, Keyes, DG, Beyer, JE, Carreon, CE, Wade, DA. et al. Results of a two-year chronic toxicity and oncogenicity study of 2,3,7,8-tetrachlorodibenzo-p-dioxin in rats. Toxicol. Appl. Pharmacol. 46, 279 (1978).

37. Gold, LS, Slone, TH, Stern, BR, Manley, NB, Ames, BN. Rodent carcinogens: Setting priorities. Science 258, 261-265 (1992).

38. Ames, BN, Gold, LS. Paracelsus to parascience: The environmental cancer distraction. Mut. Res. 447, 3-13 (2000).

39. Bohn, SK, Blomhoff, R, Paur, I. Coffee and cancer risk, epidemiological evidence, and molecular mechanisms. Mol Nutr Food Res 58, 915-930 (2014).

40. Crippa, A, Discacciati, A, Larsson, SC, Wolk, A, Orsini, N. Coffee consumption and mortality from all causes, cardiovascular disease, and cancer: a dose-response meta-analysis. Am. J. Epidemiol. 180, 763-775 (2014).

41. Keenan, JJ, Gaffney, SH, Galbraith, DA, Beatty, P, Paustenbach, DJ. Gasoline: A complex chemical mixture, or a dangerous vehicle for benzene exposure? Chemico-Biolog. Inter. 184, 293-295 (2010).

42. Laughlin, RB, Ng, J, Guard, HE. Hormesis - A response to low environmental concentrations of petroleum-hydrocarbons. Science 211, 705-707 (1981). 
43. De Nicola, E., Gallo, M, Iaccarino, M, Meric, S, Oral, R. et al., Hormetic versus toxic effects of vegetable tannin in a multitest study. Arch. Environ. Contam. Toxicol. 46, 336-344 (2004).

44. Mendoza-Figueroa T. Growth and toxicity tests on the marine nonoplanktonic algae Platymonas astetrathele G.S. West in the presence of crude oil and emulsifiers. Environ. Poll, 4, 261-282 (1973).

45. Walsh, GE, Duke, KM, Foster, RB. Algae and crustaceans as indicators of bioactivity of industrial wastes. Water Res. 16, 879-883 (1982).

46. Aube, M, Larochelle, C, Ayotte, P. Differential effects of a complex organochlorine mixture on the proliferation of breast cancer cell lines. Environ. Res. 111, 337-347 (2011).

47. Milward v. Acuity Specialty Products Groups, Inc. Civil action no. 07-11944-DPW, 2013

WL 4812425 (D. Mass. Sept. 6, 2013).

48. Whiting v. Boston Edison Co. 891 F, Supp. 12, 25 (D. Mass. 1995).

49. Sutera v. Perrier Group of America Inc. 986 F. Supp. 655, 666 (D. Mass. 1997). 
Figure 1. Functional integration of hormesis and LNT for carcinogen risk assessment; derivation of the optimal regulatory strategy

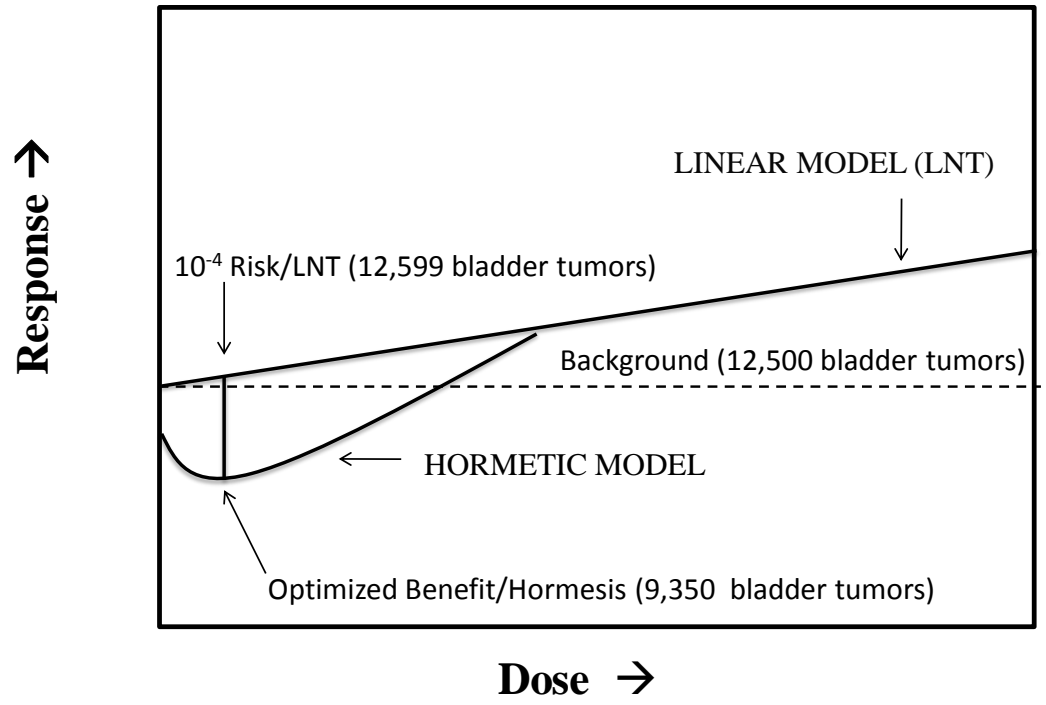

